\title{
Malformaciones anorrectales
}

Drs. Víctor Henríquez Alessandrini, ${ }^{*}$ Hemăn Jeria de Folliot** y Sergio Rojas Castro*

\begin{abstract}
S
Betweer June 70 and October 77, 35 children with anorectal malformation wene treated at the Leanor Dlascilyano Children's Surgical Hospital.

Their clinical and anatomical characteristics were analized, classifying them anconding to the concept of high or low rnalformations.

The surgical Technic employed, and their results were reviewed and the unfavourable cases were analized.

A criteria for selecting the surgical tecnic for each tipe of malformation was established.

The advantage of colsstminy and its indication in the treatment of these malfomations is commented in this papers.
\end{abstract}

El enfoque diagnóstico y terapéutico de las malformaciones anorrectales ha experimentado en los últimos años un cambio manifiesto.

De las consideraciones puramente morfológicas, se ha pasado a estimar como de fundamental importancia el aspecto funcional del problema.

En este sentido se le ha dado especial relevancia al fascículo puborrectal deI músculo elevador del ano. Este fascículo es capaz de garanti-

*Servicio de Cirugfia infantil y Ortopedia, Hospital "Leonor Mascayano", Concepeión.

Director y Jefe de Servicio. Dr. Duberli Yafiez Araya

* Profesor de Cirugia Infantil, Esc. de Medicina, U. de Concepción. zar la continencia en ausencia de los esfinteres anales, hecho frecuente en este tipo de matfonmaciones. $1,2,3,4,5$

El fasciculo pubortectal se inserta en el pubis y se dirige hacia atrás rodeando como un anillo al conducto anorrectal. ${ }^{1,6}$

Es, par todo lo anteriormente dicho, que a la tradicional clasificación de Ladd-Gross, que reconoce cuatro tipos de imperforación anal, se ha sumado una clasificación anátomo funcional que agrupa a las malformaciones anorrectales en Altas y Bajas, según sea la relación de la altura del fondo de saco con el fasciculo puborrectal, y que pone énfasis en la presencia y ubicación de la fístula. $3,7,8,9,10$ 
Malformaciones Altas o Rectales son aquellas en que el conducto anorrectal no atraviesa el fascículo puborrectal quedando su fondo de saco por encima de éste.

Malformaciones Bajas o Anales son aquellas en que el conducto atraviesa el fascículo guedando el fondo de saco por debajo de éste.

El tratamiento de estos defectos tiene como objetivos:

Establecer el transito intestinal y corregir morfológica y funcionalmente la malformación, teniendo como meta esencial la continencia. $1,3.5,9$. 10. 11

Las técnicas quirúrgicas que se emplean, están destinadas a corregir los defectos en base a los conceptos recién emitidos.

\section{MATERIAL Y METODO}

Se revisan las fichas clínicas de $3 \mathbf{5}$ nin̄os tratados en el servicio de Cirugía Infantil y Ortopectia del Hospital Leonor Mascayano, de Concepción, entre junio de 1970 y octubre de 1977. Se analizan los diversos tipos de procedimientos quirúrgicos empleados y se evaluan sus resultados.

Figura 1

\section{INCIDENCIA POR SEXD}

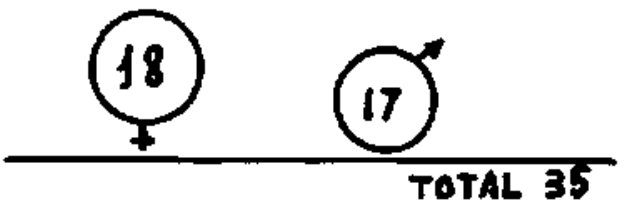

Sexo.

De los 35 casos, 18 fueron mujeres y 17 hombres.

Peso de nacimiento.

Está consignado en 26 casos, de los cuales 20 están solre los $2.500 \mathrm{gr}$.

Figura 2

\section{PESO NACIMIENTO CONSIGNADO} EN 26 CASOS

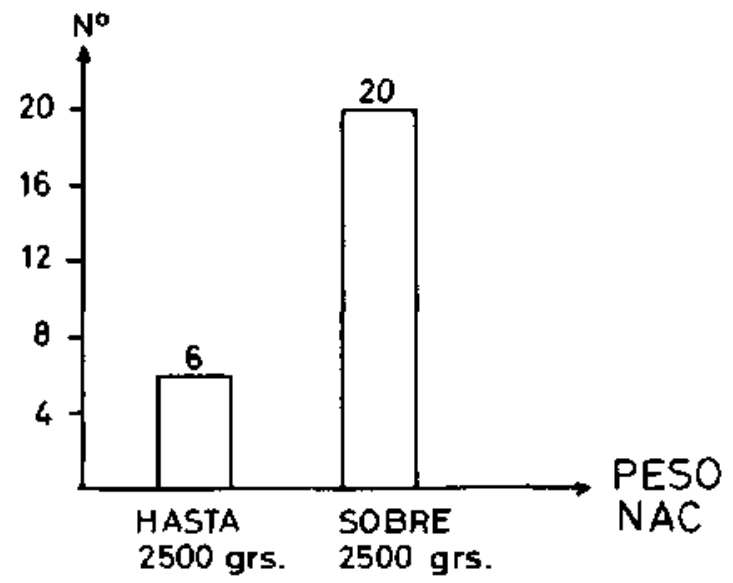

\section{Procedencia.}

15 casos procedian de Concepción y Talcahuano. Los 20 restantes provenian de otras localidades de la octava región, lo que explica la falta de autecedentes en algunos casos

Figura 3

\section{PROCEDENCIA}

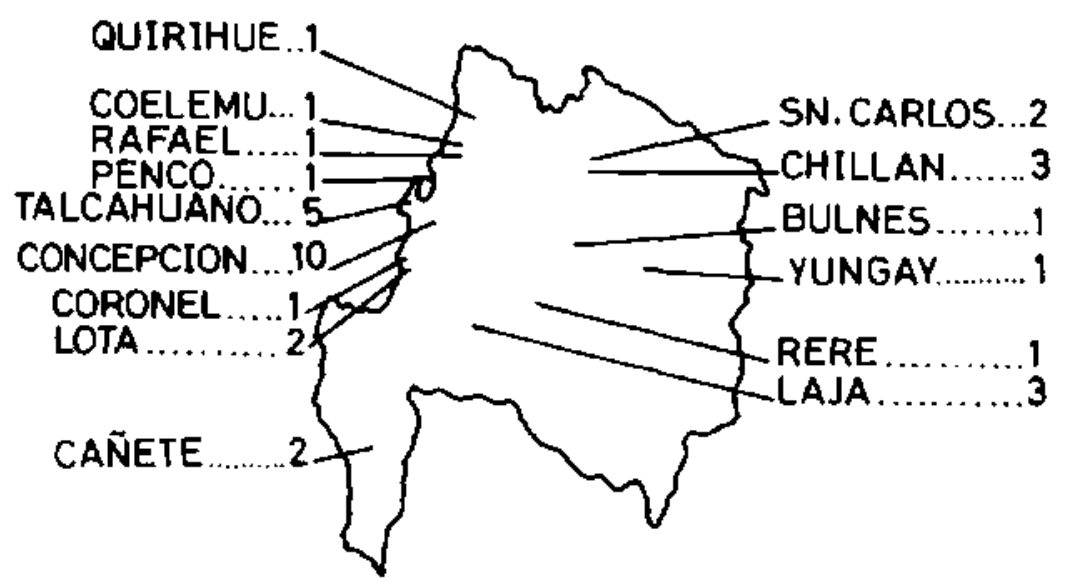


Antecedentes obstétricos.

Están consignados en 29 casos. En ninguno, existen antecedentes teratogénicos manifiestos. En 23 casos el parto fue eutócico, y los 6 casos de parto distócica se desglosan en 3 cesáreas 2 pautos en podálica y l parto prenuaturo.

\section{Figura 4}

\section{ANTECEOENTES OBSTETRICOS}

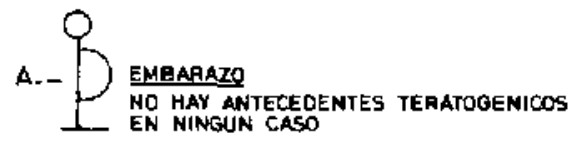

B. -PARTO
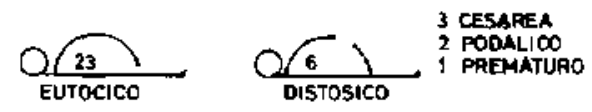

Presencia de fistula.

En 31 de los 35 casos se constatô la presencia de fístula $(88,5 \%)$.

En 17 de las 18 mujeres había fístula $(94,4 \%)$. La única excepciôn es una nin̄a mongólica nacida en octubre de este año, en que se hizo el diagnóstico de Atresia rectal.

EI 3 de los 17 hombres no se comprobó fístula (21,4\%), correspondiendo a 2 casos de Agenesia anal y 1 caso de Membrana anal imperforada.

\section{Figura 5}

PRESENCIA DE FISTULA

\begin{tabular}{|l|c|c|c|}
\cline { 2 - 4 } \multicolumn{1}{c|}{} & $\begin{array}{c}\text { MUJERES } \\
N^{\circ}\end{array}$ & $\begin{array}{c}\text { MOMBRES } \\
\text { No }^{\circ}\end{array}$ & TOTAL \\
\hline CON FISTULA * & 17 & 14 & 31 \\
\hline SIN FISTULA & 1 & 3 & 4 \\
\hline T O T A L & 18 & 17 & 35 \\
\hline
\end{tabular}

* Ineluye Ano Ectópico

Figura 6

\section{TIPO DE MALFORMACIONES} ENCONTRADAS

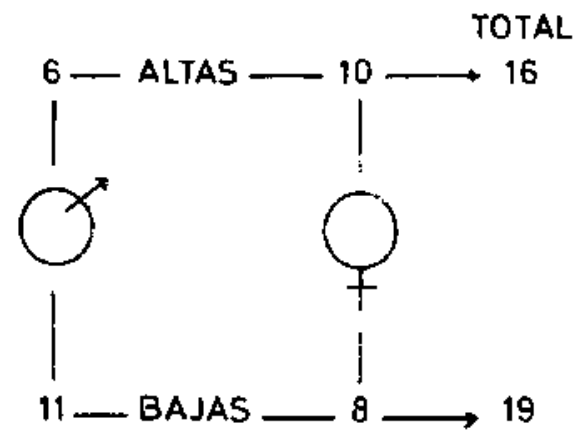

Tipos de malformaciones encontradas.

De los 35 casos 16 correspondieron a Malformaciones altas y 19 a bajas.

En los hombres fueron más frecuentes las bajas, $y$ en las mujeres predominaron las altas.

Figura 7

\section{MALFORMACIONES MAS FRECUENTES}

MUJERES:

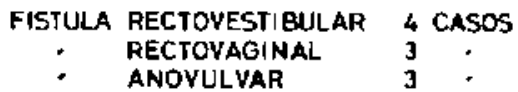

HOMERES

$\begin{array}{clll}\text { FISTULA ANOPERINEAL } & \mathbf{6} & \cdot \\ \cdot & \text { RECTOVESICAL } & \mathbf{3} & . \\ \cdot & \text { RECTOURINARIA } & \mathbf{3}\end{array}$

En los hombres los tipos de malformaciones más frecuentes fueron: Fistula anoperineal 6 casos, Fístula rectovesical 3 casos, Fístula recto urinaria 3 casos.

En las mujeres los tipos más frecuentes fueron: Fístula rectovestibular 4 casos, Fistula rectovaginal 3 casos y Fístula anovulvar 3 casos.

\section{Edad de diagnostico.}

De los 11 casos sin Fístula o con Fístula insuficiente, 9 fueron diagnosticados antes de las 48 horas y 2 después; uno de ellos era de procedencia rural, el otro nació en una clínica de Concepción.

De los 24 casos con fístula suficiente sólo 12 fieron diagnosticados antes de las 48 horas. En los demás pacientes el diagnóstico se hizo antes de un mes de edad, en 8 y después en 4.

\section{Anomalias asociulas.}

Su presencia fue evidente en 16 casos. No se buscaron intencionadamente, sino que fueron un hal lazgo clinico o quírúryico.

Las más frecuentes fiueron las digestivas con 5 casos, de los cuales 4 correspondieron a enfermedad de Hirsclsprung. Siguiendo en frecuencia se encontró 3 Malformaciones Urinarias, 2 de Colunna y 2 Cardíacas. En 2 niñas habian malformaciones múltipies (urinarias, esqueléticas, cardiacas, neurológicas) y finalmente, uno de nuestros pacientes tenía Artrogriposis y otro Mongolismo. 


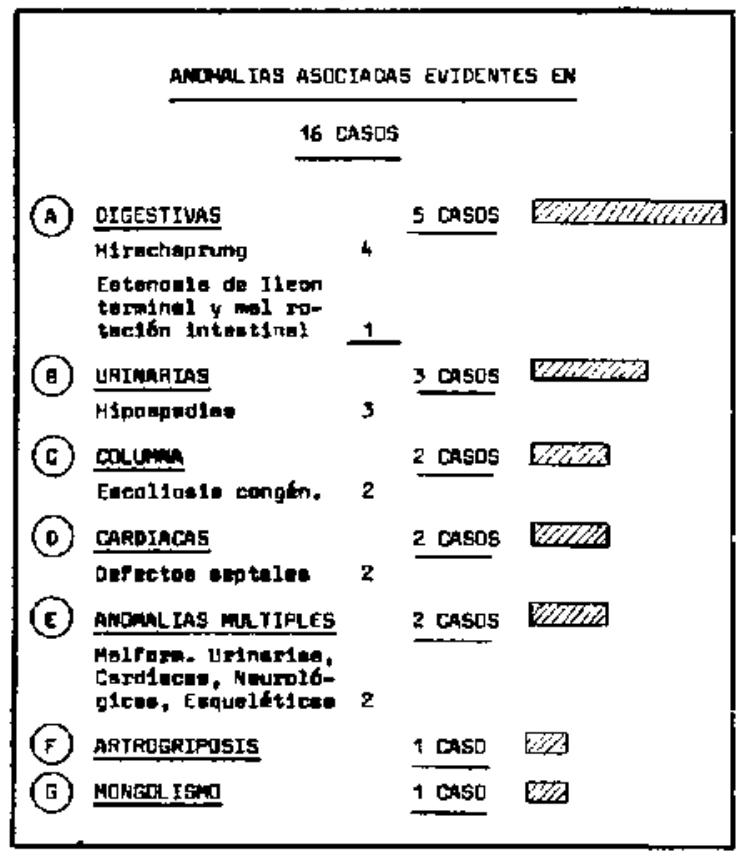

Técnicas quinirgicas empleadas: "cut-back".

Es similar a una episiotomía media. Consiste en abrir la fístula anoperineal (a veces ano vulvar 0 ano vestibular) hasta alcanzar la pared posterior del fondo de saco. Queda expuesta la mucosa de la fístula, $y$ la del fondo de saco, la primera se reseca y la segunda se sutura a la piel. $3,4,5,7,8,10$

Anoplastía.

Operación por vía perineal, usada sólo en malformaciones bajas mediante la cual se reseca la tistula, si ella existe, $y$ se lleva el fondo de saco a la foseta anal, abriéndolo y suturando mucosa a piel. $3,4,5,9,10,11$

\section{Descenso abdominoperineal.}

Es la operación definitiva para las malformaciones altas. Consiste en abordar por vía abdominal e] fondo de saco, liberarlo, resecar la fístula y descenderlo hasta la foseta anal, pasando por dentro del anillo pubo rectal y a través de un túnel Iabrado desde el periné. $1,3,4,5,9,10,11$ "

Tratamientos quirírgicos efectuados.

Se realizaron 45 intervenciones quirúrgicas:

- "Cut-Back"

- Anoplastía

- Colostomías

- Descenso abdomino perineales

5

Evolución y resultados de los procedimientos quirürgicos efectuados.

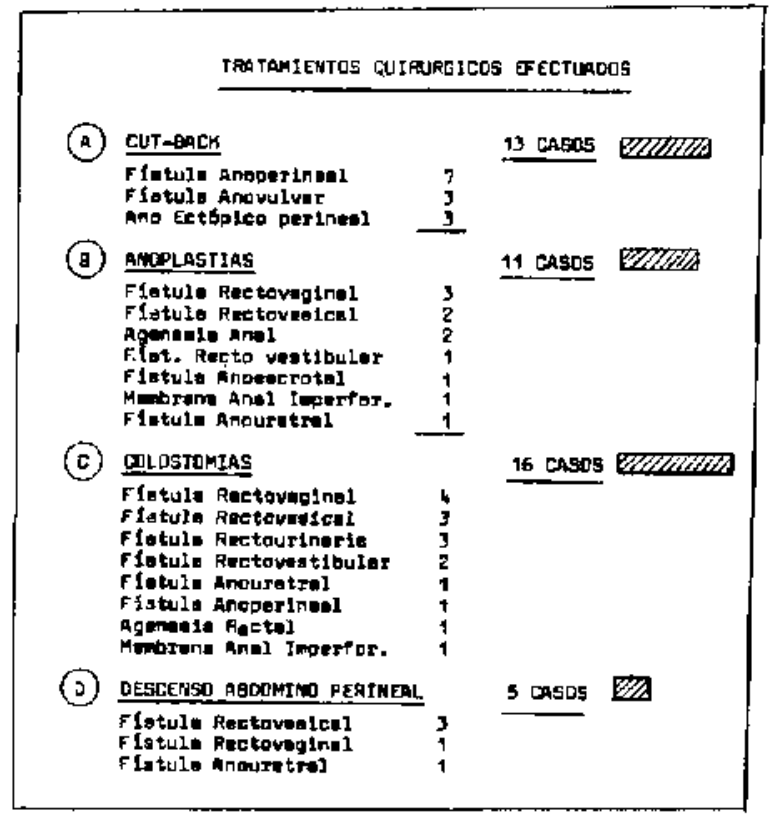

Cut-Back. Se obtuvo buen resultado funcional, vale decir sin estenosis, en 11 de los 13 casos. En los 2 casos restantes los niños evolucionaron con constipación, lo que en todo caso no puede ser attribuido a la intervención. En uno de estos niños habia enfermedad de Hirschprung.

El resultado estétíco fue malo en 2 mujercitas, una de ellas con fístula anovulvar, $y$ la otra con ano ectúpico vulvar. En ambos casos la intervención no dejó separación perineal entre vulva y ano. Uno de ellos fue corregido mediante una plastía perinéal dos años después del "Cut-Back", con excelente resultado.

Anoplastías: Tres niños evolucionaron sin complicaciones. Seis casos hicieron Estenosis Anal, la cual se detectó entre 10 días y 2 meses después de la intervención. En ninguno de los casos se había hecho dilataciones periódicas después de la Anoplastía, debido en parte, a que 4 de los 6 niños no eran de Concepción.

Todos los casos de Estenosis fueron tratados posteriormente con dilataciones, y en los casos en que el hecho se consignó, el resultado final es bueno.

La Fistula Vesicoperineal iatrogénica se debió a una Anoplastia mal indicada, en un Hospital periférico, a un recién nacido con fístula rectovesical. 
La muerte se produjo en un recién racido de 22 dias, a quien se le hizo una Anoplastia a los 8 días de edizl. Fialleció por Sepsis debido a Peritonitis por rotura espontánea del íleon temninal, que preseritaba nua Estenosis congénita.

Colostomia. Once casos evolucionaron sin complicación. En uno de ellos se produjo Prolapso, en otro hubo Prolapso y Hemorragia. Un niño presentó Prolapsos a repetición y finalmente evisceración 6 meses después de la Colostomia. Hubo epitelización de los estomas en un caso. El fallecimiento se produjo en an niño de 1 mes y 7 clías de edad, 4 días después de la Colostoníí. El cuddro clínico fue compatible con una Sepsis por Peritonitis.

En todos los casos, salvo uno en que no está consignado, la Colostomia fue hecha en el colon izquierdo (transverso izcquierdo 6 casos, Angulo esplénico 3 casas, Sigmoides 6).

No se encontró relación entre el nivel de la Colostomía y la aparición de complicaciones.

Desce'nso abdominoperineal. La técnica usada fice lit preconizada por Gross y Benson.

Una riña evolucionó sin complicaciones.

En un caso se produjo Estenosis anal moderadiat que se corrigió con dilataciones.

En un caso hubo Prolapso rectal irreductible, que se corrigió quirúrgicamente.

En un caso huho Evisceración 5 días después de la intervención, evolucionando luego satisfactoriamente.

El fallecimiento se produjo en um niròo de $\mathbf{1}$ año 9 meses como consecuencia de septicmia por peritonitis originada en una perforación quiningica intraperitoneal del asa descendida, clue a pesar de haber sido suturada cuidadosamente, sufrió una dehiscencia. Cabe destacar que se trataba del lactante con unit Fistula vesico-perimeal iatrogénica debida a una Anoplastia mal indicadia y mal efectuada que dejó un terreno quinirgico particularmente difícil.

Respecto a la continencia no se puede tabular el resultado, ya (pue de los 5 casos, 1 lalleció, otro tiene anonalias de columna sacra, cuyo componente neurológico es causa de incontinencia, y los 3 restantes no han vuelto a control.

fiesumen de fallecidos.

Hubo un total de 5 fallecidos, todos varones.

La edad osciló entre los 22 días y 1 año 9 meses.

La causa del deceso determinada clínica o quirúrgicamente en 4 casos fue Sepsis por Peritoni- tis. En el ciaso restarite no está consignada por extravío de parte de la ticha clínica.

Respecto a la relación entre el acto operatorio y la causa de la nuerte, existe en 2 casos: el niño que fálleció 4 días después de una Colostomía, y otro fatlecido, después del descenso abdomino perineal.

En dos casos no hay relación entre la intervención quirúrgica y la causa de la muerte: 1 niño con Anoplastia, falleció a raíz de la rotara espontanea del íleon ternuinal; I niño en quien se hizo Colostonía a los 3 días de edad, evolucionó con desnutrición importante, infección urinaria crónica, y prolapsos à repetición difíciles de reducis, finalmente se evisceró at lon 6 meses, haciendo un cuadro clínico compatible con Peritonitis $y$ Sepsis.

\section{Figura 10}

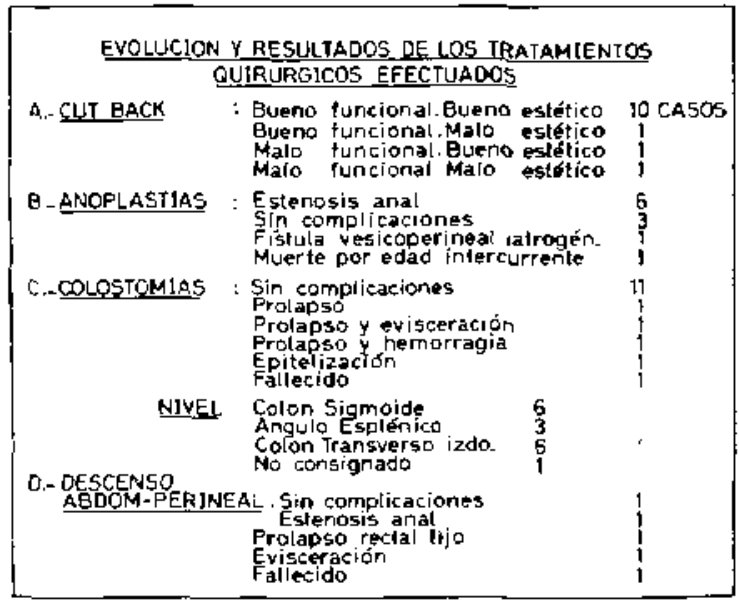

Tabla 11

RELACION ENTRE ACTO OPERATORIO $\checkmark$ CAUSA DE MUERTE

a) RELACIONADA CON ACTO OPERATORIO:

$\begin{array}{ll}\text { - Post Colostomía } & \text { 1 Caso } \\ \text { - Descenso abdómino perineal } & \frac{1}{2 \text { Casos }}\end{array}$

b) NO RELACIONADA CON ACTO OPERATORIO:

-Perforación espontánea. Estenosis
Ileon terminal. 1 Caso
-Desnutrición. Infección urinaria cron.
Prolapsos a repelición-Evisceración 1
TOTAL Casos

\section{COMENTARIO}

Lals malfonmaciones anorrectales son relativamente frecuentes y se asocian en un alto porcentaje con otras malformaciones congénitzs. Su diag- 
nóstico debe ser precoz, mediante un buen examen del Recién Nacido, y preciso en lo referente a la presencia de fístula, altura del fondo de saco, y malfomaciones asociadas. Por su alta incidencia la fístula debe ser buscada detenidamente.

No debe ocurrir el diagnóstico tardío en un niño nacido en un Centro Asistencial.

Para que el tratamiento cumpla con los objetivos de establecimiento del tránsito intestinal y corrección mortológica y funcional de ta malformación, se debe tener un amplio conocimiento anátomo funcional de la región, por lo gue estos niños deben ser tratados en Centros Especializados.

La relativa importancia del estinter anal extemn? en la continencia, queda en evidencia en los niños sometidos a "Cut-Back" en los cuales el esfínter extemo se secciona y son continentes y en el caso de dos niñas con fístuka rectovaginal operadas después de los 7 an̄os y que eran y siguen siendo continentes.

Estimamos que las malformaciones Bajas son susceptibles de ser corregidas definitivamente, en forma precoz dentro de las primeras semanas de vida.

Las malformaciones Altas deben ser corregidas en forma diferida, $y$ la operación definitiva debe ser precedida de una Colostomía transversa, todo ello por razones de tipo técnico.

En cuanto a las técnicas quirúrgicas, el "CutBack" es una muy buena intervención para la corrección de las fístulas ano perineales. En la fistula anovulvar estaría indicado cuando ésta es, o se hace insuficiente, ya que, en general, preferimos mantener este tipo de Fístula, con dilataciones periódícas hasta el año y medio de vida, edad en que se puede corregir mediante una Anoplastía.

La Anoplastia es una técnica sencilla, pero debe ser hecha por un Cirujano experimentado, prueba de ello es el niño a quien se abocó la vejiga al periné. El uso de la Colostomía Sigmoidea previa, aunque implica dos tiempos operatorios adicionales, deriva el tránsito intestinal, disminuye el riesgo de infección, permite la cicatrización primaria, y por lo tanto previene de las dehiscencias $y$ retracciones secundarias.

Es imprescindible efectuar dilataciones periódicas postoperatorias precoces y por un tiempo prolongado.

En las malformaciones Altas la Colostonía es la indicación precisa $y$ necesaria ante una urgencia como es establecer e] tránsito intestinal, ya que permite cumplir con este objetivo en toma rápida, sencilla y eficiente, se hace en el colon transverso izquierdo, no ofrece dificultades para su manejo posterior, faciliti el estudio radiológico, disminuye el riesgo de infección urinaria y deja longitud de intestino suficiente para realizar (un desceriso abdómino perineal.

El descenso abdómino perineal requiere elementos, experiencia, observación rigurosa de los cuidados preoperatorios, especialmente el vacia* miento del asa distal, y una técnica acuciosa durante el acto operatorio a fin de evitar complicaciones.

\section{RESUMEN}

1. Se revisan las fichas clínicas de 35 nin̄os portadores de malformaciones anorrectales, tratados en el Servicio de Cingía Infantil y Ortopedia, Hospital Leonor Mascayano, de Concepción, entre junio de 1970 y octubre de 1977.

2. Se establecen sus caracteristicas clinicas y los aspectos epidemiológicos más fundanentales.

3. Se estudian los diferentes tipos de malfomaciones encontradas, clasificándolas de acuerdo a los conceptos actuales, en Altas y Bajas, enfatizando la presencia y ubicación de la fistula cuya incidencia en nuestra serie fue elevada ( 31 casos).

4. Se estudian las técnicas quirúrgicas utilizadas en el tratamiento de cada una de ellas y sus respectivos resultados.

5. Se analizan los malos resultados.

6. Se señalan, de acuerdo a la experiencia del Servicio, las indicaciones de cada una de las técnicas quirürgicas analizadas, de acuerdo al tipo de malformación.

7. Se comentan las ventajas de la Colostomía, y sus indicaciones en el tratamiento de las malformaciones Altas y Bajas.

\section{REFERENCIAS}

1 Stephe'ss, D. Embryologic anid functional Aspects of Imperforate Anus. Surg. Clin, of N.A. 30-919, 1970

${ }^{2}$ Puri, $P_{\text {, Nixur, }} \boldsymbol{H}$. The Internal Ausal Sphincter iu Translevator (Low) Anal Anomtalies. J. Pediat. Surg. 11: 553, 1976.

'Del fifo, A. Malformaciones Anortectales en el Niño. Cnuader. nos Chílenos de Cirugra. 20): 146, 1976.

${ }^{4}$ Vixun, H., Puri, P. The results of Treatment of Anorectal Anomalles: A Thirteen to Twenty Year Follow-Up. J. Pediat. Surg. 12: 27,1977

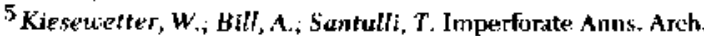
Surg. 111:518, 1976

6 Testut, L.; Letarjet, A. Anatomia Humana (Barcelona: Salvat Editores S.A., 1965). 
${ }^{7}$ Santulti, T.; Schutlinger, $J_{\text {; }}$ Amoury, R. Maltomaciones de Ano y Recto. Clin. Quir. de N.A. 1253, Oct. 1965.

${ }^{2}$ Stumilli, $T$.; Kiessenuetter, $W$; Bill, $d$. Anorectal Anonalies: A Suggested lntemational Classification. J. Pediat. Surg. 5: $28 \mathrm{~L}, 1970$.

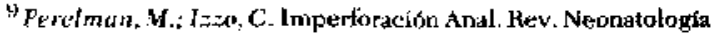
$1: 4,1971$.
${ }^{10}$ Adkins, $J . ;$ Kiesewetter, $W$. Ano Imperforado. Clin, Quir. de N. A. 56: 379, 1976

1 I Singh, M.; Haddudin, A.; Zachary, R.; Pilling, D. Renal Tract Disease in Imperforate Anus. J. Pediat. Sury. 9; 197, 1974.

i2 Bension, C. et al. Cirugga Infantil (Barcelona: Salvat Editores S.A., 1967). 